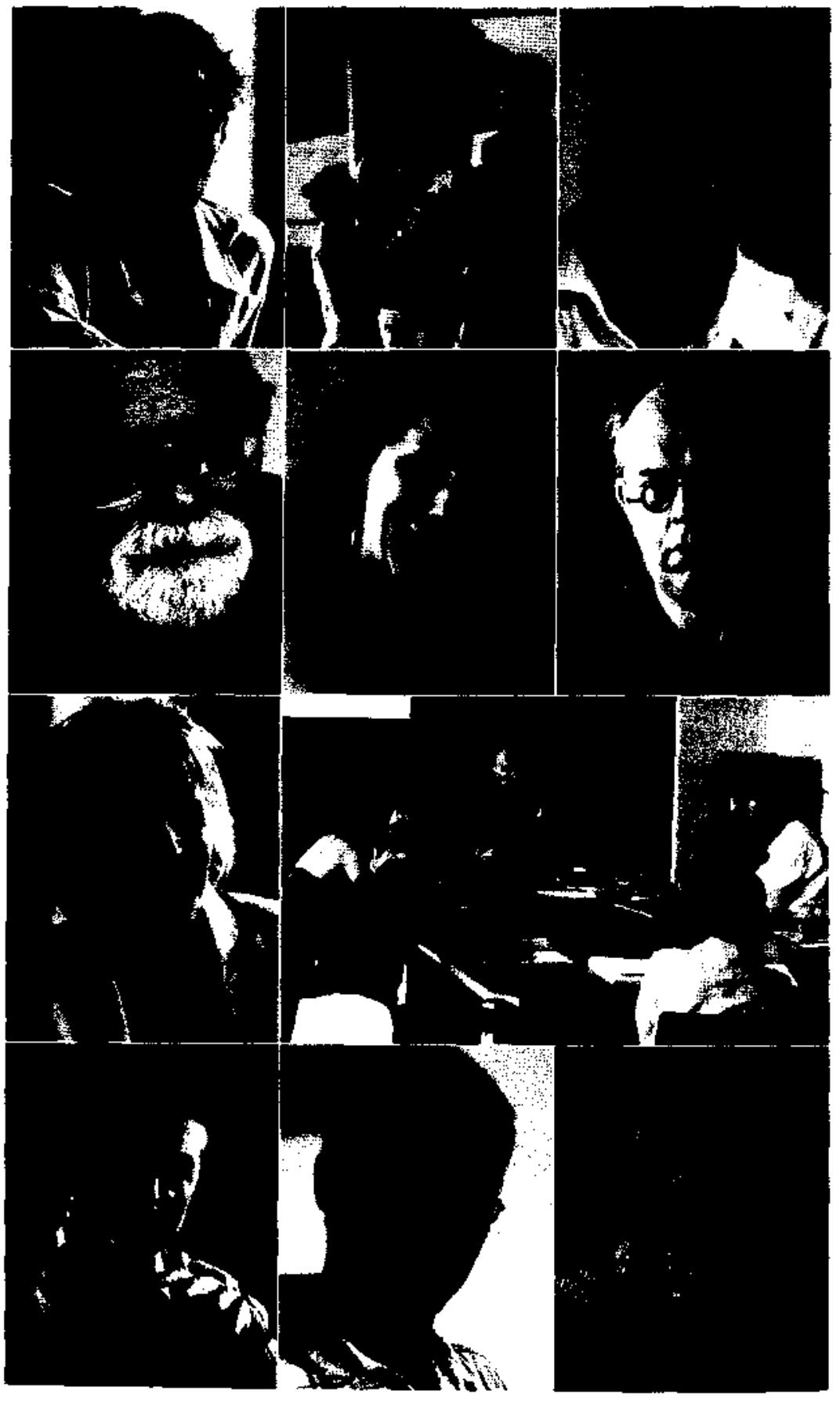




\title{
14
}

\section{What Are the Brain Mechanisms Underlying Syntactic Operations?}

\author{
Anna Fedor, Csaba Pléh, Jens Brauer, David Caplan, \\ Angela D. Friederici, Balázs Gulyás, Peter Hagoort, \\ Tatjana Nazir, and Wolf Singer
}

\begin{abstract}
This chapter summarizes the extensive discussions that took place during the Forum as well as in the subsequent months thereafter. It assesses current understanding of the neuronal mechanisms that underlie syntactic structure and processing.... It is posited that to understand the neurobiology of syntax, it might be worthwhile to shift the balance from comprehension to syntactic encoding in language production
\end{abstract}

\section{The Main Questions}

The focal question posed to our group can be answered on many descriptive levels. At the finest-grained level are processes that store and activate syntactic representations in neuronal networks. Here, relevant variables are the frequency of spikes, the temporal structure of spike sequences, changes in sensitivity and number of synapses, and modifications of excitability. To date, however, little is known about the physiological basis for syntactic processing at this cellular level. In our discussions we therefore concentrated on relating syntactic representations and processes to larger-scale neural features, such as event-related potentials (ERPs) and areas of the brain determined anatomically or functionally.

It is important to realize why issues related to the "localization" of cognitive skills in the brain, including the processing of language, are hotly debated. These issues have a long history in neuroscience, and the answers given were always dependent on the original assumptions, the methodology, and methods 
of the investigations. Nowadays it cannot be denied that the textbook notion of "Broca's area for syntax and Wernicke's area for semantics" is outdated. Localization of components of language, including syntax, shows variation both across and within individuals; the latter indicates that the problem has a strong ontogenetic component as well. The enormous plasticity of the developing human brain (as shown by recovered language after lesions early in life) demonstrates that the crucial involvement of Broca's area in syntactical processing in most people cannot be a genetically hardwired, rigid condition. It seems more correct to say that some areas of the normally developing human brain are more prone (in a quantitative sense) to host and process different components of language than others. This "hospitality" may be due to the particular features of the local microanatomy/physiology or connectivity to other areas, or both. Data support both alternatives. Ultimately, what we need is a detailed neuronal model of how syntactic operations can be implemented as well as how such neuronal structures could have been rigged by genetic influence. Apes do not talk and acquire syntax; by comparison, humans with even low IQs are linguistic geniuses. We need to gain an understanding of the neurobiological and correlated genetic changes in evolution; we are slowly but surely approaching this target. This chapter reports on the current level of understanding.

\section{Syntactic Structure and Processing}

In its essence, syntax is a means by which elements are arbitrarily combined; in the case of language, the elements are semantically interpreted symbols. The syntax of natural languages has a number of properties that have guided the study of its evolution and neurological basis. Several pertain to the nature of the meanings that it encodes; others to the forms of syntactic representations.

Three important features of semantic meanings encoded by syntactic relations are:

1. Meaningful symbols that are combined syntactically are both referential (reflected in, e.g., content words) and logical/formal (reflected in, e.g., function words and affixes).

2. Meanings conveyed by combinations of these symbolic elements, collectively referred to as propositional and discourse-level meanings, constitute a restricted set of semantic values and include the relation between items and their properties (modification and quantification), the participant structure of events (thematic roles), temporal and aspectual features of events, illocutionary force, discourse prominence, as well as others.

3. Propositional meanings can be combined with the referential meanings of individual items, as in the modification of referential elements by 
propositions using relative clauses (e.g., The boy who is wearing the blue shirt is my brother). Propositions can also be combined with other propositions, as in complementation (e.g., John believes that carrots are a good source of vitamins). Meanings are therefore compositional, productive, and recursive.

The syntactic relations between constituents that determine aspects of propositional and discourse meaning have been the subject matter of intense inquiry on the part of linguists since the seminal work of Noam Chomsky in the 1950s. Models of these relations differ widely. The latest version of Chomsky's theory, for instance, maintains that binary branching nodes create hierarchical structures in which nodes "merge" "externally" to create local phrase markers that assign certain semantic values. This aspect of the syntax is the basis for the compositional, productive, and recursive features mentioned above. Second, in this model, constituents "merge" "internally" to create complex phrase markers, subject to language-specific and language-universal constraints (Chomsky 1995, 2004, 2006). Semantic relations between constituents are determined and constrained by particular asymmetrical dominance relations between nodes in these phrase markers ("c-command"). Other linguists view syntax differently. Goldberg $(1995,2006)$ and Croft $(2001)$, for example, argue that the syntactic structure of a sentence is a direct reflection of its surface form, and that propositional meanings are determined by the "constructions" of the syntax (see Tallerman et al., this volume).

Regardless of how the syntactic structures that determine propositional and discourse meanings are represented, they must capture the fact that formmeaning relations are complex. Take, for example, two of the central features of syntax: hierarchical structures and distant dependencies. These features can be distinguished from each other. One can easily compose sentences with distant dependencies, where the distance between the connected words is made wider by inserting adverbs. Thus, in empirical studies, it is very important to ensure that it is indeed complexity per se (hierarchical structure including distant dependencies vs. linear structure including local dependencies only) and not merely greater distance between constituents that makes the understanding of one sentence more difficult than another. If the distance between constituents is too large (e.g., in the case of a very long sentence), it may be difficult to judge whether the sentence is grammatically correct, even if the structure is simple. It is therefore crucial to disentangle empirically the factors of syntactic complexity and working memory through a design that varies these two factors independently whenever possible (Makuuchi et al. 2009); see Figure 14.1 for examples.

For processing long-distance dependencies, a system is needed that carries information over time. In the human brain, the prefrontal cortex may be suitable to this task because of its involvement in working memory. Its ability to support short-term functions is known from neuropsychological studies in 
Peter wusste, dass...

Peter knew that...
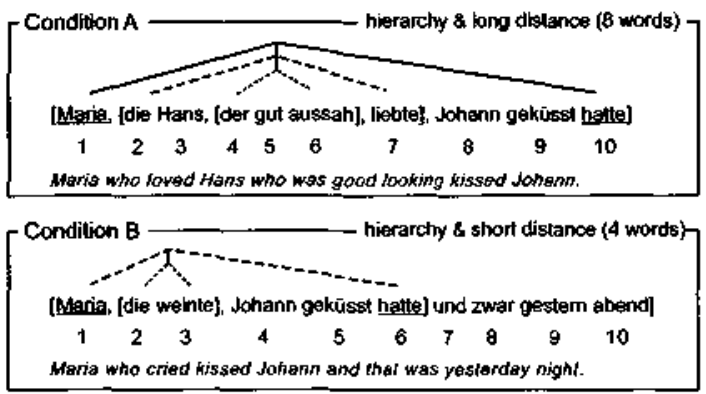

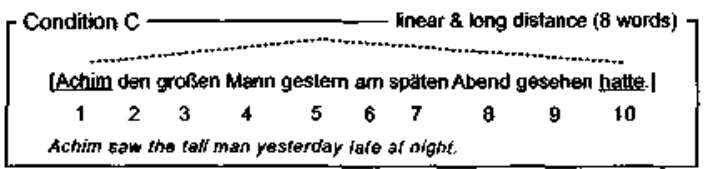

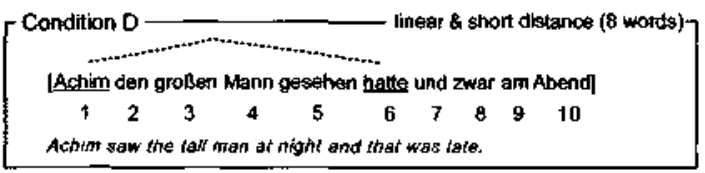

Figure 14.1 Example sentences for the four possible combinations of linear/hierarchical structure and short-/long-distance dependencies.

human subjects and animal experiments. Understanding the neural mechanism for processing hierarchical structures in sentences is, however, a much harder problem and, recent pioneering studies in this field notwithstanding, there are only speculative ideas about its implementation in the brain. A step toward understanding this issue are findings from brain imaging studies, which suggest that the effects of long-distance dependencies and those of hierarchical structures are processed in separable, but closely interacting areas in the inferior frontal gyrus (IFG; see Figure 14,2) (Makuuchi et al. 2009; Santi and Grodzinsky 2007b).

The example above demonstrates potential interactions between linguists, psychologists, and cognitive neuroscientists. In general, linguists and psychologists can provide theories that constrain the hypothesis space regarding syntactic structures and their processing, presenting testable predictions; neuroscientists can provide empirical evidence that constrains the theory space. Experimental evidence could help linguists decide between competing models of structure and processing. Neuroscientists, in turn, need from linguistics a certain level of generalization across the different versions of linguistic theoretical models. This would help define aspects of complexity and structure for translation into experimental manipulations aimed at identifying biological consequences. 


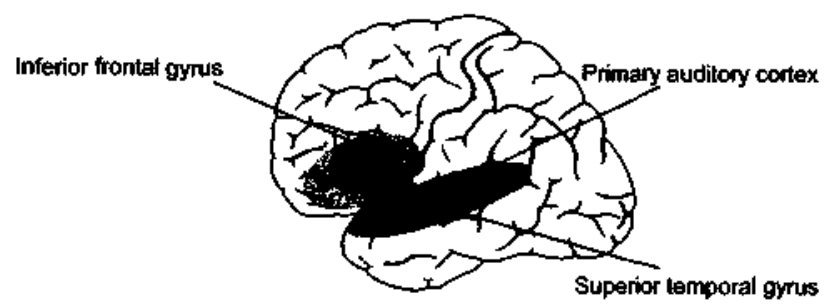

Figure 14.2 Left sagittal view of the human brain. The shaded areas indicate the main language-relevant perisylvian regions in the left hemisphere (LH). Broca's area proper (Broca 1863) comprises Brodmant area (BA) 44 and BA 45 of the IFG (Amunts et al. 1999), Further frontal areas (light gray), also involved in some language processes, are BA 47 of the IFG and the precentral gyrus (BA 6), and it has been suggested to label all four areas together as Broca's area extended (Hagoort 2005). The shaded region around 22 and 42 depicts the superior temporal cortex with the prinary auditory cortex (dark gray) in its mid-portion.

To illustrate such an interdisciplinary interaction, consider the syntactic phenomenon that many linguistic theories specify as the movement or displacement property in many natural languages; that is, elements are often pronounced in positions distinct from those in which they are interpreted. For example, in a question like

(1) Which book do you think I should read?

the phrase which book must be interpreted as a thematic argument of the verb read. It, however, is not in the canonical object position adjacent to the verb as in I should read this book. Instead, it has been displaced to the front of the sentence and, in fact, can be indefinitely far away from its thematic position. Thus, the grammar and the parser must be able to relate two positions which can be quite distant in the tree structure. One may think that this operation has a certain computational cost which brain imaging studies may be able to highlight, in terms of accrued activation of the neural structures which are involved. As movement can be seen as a sub-case of Merge, the fundamental recursive structure-building mechanism, it can be applied several times in a structure. One might entertain the working hypothesis that the number of applications of movement provides a rough measure of complexity (Jakubowicz, unpublished). This would be difficult, however, to substantiate, because it is likely that different types of movement have different computational costs; for instance, the computational cost of some very local movements (e.g., head movement of the verb to the inflectional system or to the complementizer in verb second language) may be negligible if compared to the cost of long-distance phrasal movements. We can avoid these complications, if we restrict our attention to phrasal movements.

An important property of long-distance movement is that, in theory, it typically takes place through a number of successive local steps in compliance with 
the requirements of locality principles. There is strong and varied linguistic evidence suggesting that the movement of our sample sentence in (1) takes place in at least two steps: (a) from the thematic argument position to the embedded complementizer system, and (b) from the embedded to the main complementizer system. In (2), the blanks indicate the two "traces" of movement:

(2) Which book do you think that [I should read ]]

To test this hypothesis empirically, it would be interesting to compare this movement in terms of computational cost with the case in which two distinct constituents have moved once. A possible approximation of this in English may be a simple clause in which one verbal complement is passivized and the other is moved:

(3) To whom was the book given by John?

Another possibility might be to investigate this phenomenon in languages with scrambling (Germanic) or cliticization (Romance) structures in which two verbal complements are allowed to take free-word order to a certain degree, or where pronouns are moved to a higher position in the tree structure of the sentence. Testing scrambling in German subjects, Friederici et al. (2006a) found Broca's area to increase its activation parametrically as a function of objectnoun phrases moved (scrambled) in front of the subject-noun phrase.

Movement is only a one-level complexity problem. If the sheer number of movements provides a rough measure of complexity, one would expect, if the hypothesis is correct, that two successive movements of the same element, as in (2), would roughly correspond to two local movements of two distinct constituents that have each moved once, as in (3). Finding similar neural effects of (2) and (3) relative to an appropriate control would support the view that movement occurs through a series of local steps, and demonstrates an important case where linguists and neuroscientists could benefit from each others' point of view.

\section{Neural Organization for Processing Syntax in the First Spoken Languages of Adults}

\section{Neural Codes for Syntax}

How are syntactic categories and their relationships coded in terms of physiological properties of neural tissue? A leading hypothesis is that they are related to the frequency of neuronal firing patterns and their correlations across space and time. Some data, collected during neurosurgical procedures, are available on extracellular recordings of single neurons in response to language stimuli; more data exists on such responses in subdural electrode grids used in preparation for resections (Quian Quiroga et al. 2008; Mormann et al. 2008). These 
studies raise the possibility of a neural code for syntax, but further research is needed to determine if there is any specificity for syntactic processes on this elementary level.

\section{Lateralization}

Since the early studies by Paul Broca (1863), language has served as an example of functional cortical lateralization in the human brain. The common notion of language lateralization to the LH does not, however, imply that the right hemisphere $(\mathrm{RH})$ is not involved in language processing. Instead, it refers generally to a stronger activation in $\mathrm{LH}$ than in $\mathrm{RH}$ and to differences in the functions that the two hemispheres perform at each level of language processing. This has been studied fairly extensively with respect to auditory and phonological processing. One model of functional asymmetry in the auditory cortex differentiates the processing on a basic feature level of auditory information to the two hemispheres. In this model, temporal information is processed primarily in the $\mathrm{LH}$ and spectral information primarily in the $\mathrm{RH}$ (Zatorre and Belin 2001). The role of the two hemispheres in syntactic processing remains uncertain. Some studies have found bilateral activation associated with syntactic contrasts (e.g., Just et al. 1996; Cooke et al. 2002). Other models of language processing suggest a specialization of the perisylvian cortex of the LH for processing semantic and syntactic information (Friederici 2002), while the perisylvian cortex of the $\mathrm{RH}$, in particular the posterior superior temporal gyrus (STG) and the frontal operculum, is regarded as responsible for the processing of prosodic information (Meyer et al. 2002; Meyer et al. 2004; Zatorte et al. 2002; Friederici and Alter 2004).

Consonant with functional hemispheric specialization, structural hemispheric asymmetries were found in language-related areas of, for example, the planum temporale in the perisylvian cortex (Geschwind and Levitsky 1968) and in the organization of intrinsic connectivity (Galuske et al. 2000). Amunts et al. (1999) reported a left-larger-right asymmetry for BA 44. The relation of these specific features of brain structure to aspects of syntactic processing remains unknown.

Hemispheric specialization is not unique to language. Besides the speech and language domain, cortical laterality has been reported for a variety of sensorimotor functions such as handedness or visual processing and other cognitive functions such as memory (Gainotti 2007; Habib et al. 2003). Morphological asymmetries have also been observed for cortical areas not related to language processing, e.g., the primary motor cortex (Amunts et al. 2000). From a comparative perspective, the morphological asymmetry of the planum temporale does not seem to be exclusively human; it has been found in nonhuman primates (Gannon et al. 1998). Functional hemispheric specialization is also not uniquely human; it has been observed in the auditory cortex of songbirds (George et al. 2004). Despite the fact that we know very little about the cortical 
organization of the brain of some other species, hemispheric organization can be inferred from lateral bias. Lateral bias (as opposed to cortical lateralization), referring to afferent or efferent bias (e.g., hand or eye preference), can be assessed behaviorally, and has been discovered for a variety of nonhuman species, including chicks, rats, and primates (Denenberg 2005; Hopkins and Cantalupo 2008; Rogers 2008).

Given its ubiquity among species and its very early presence in development, laterality appears to be a phylogenetically old phenomenon. It may underlie homologous phenomena across species or constitute a convergent characteristic that has been repeatedly favored in natural selection. However, the evolutionary advantage conferred by hemispheric specialization remains unknown, and the extent to which functional and/or structural hemispheric specialization might have promoted the evolution of language and syntax remains an open issue.

\section{Regional Specialization}

There is a general consensus that cognitive operations engage cortical macronetworks consisting of activated neuronal populations in various brain regions. To perform a certain cognitive task successfully, the involvement of a number of activated neuronal assemblies is required. A widespread view holds that a core network performs a set of operations that occur under all conditions of performance, and that this core network is complemented by other activated neuronal populations that map its products onto input and output systems, memory, decision making processes, etc. Different nonlinguistic systems are engaged as a function of varying modalities or stimulus and task conditions, and their involvement affects efficiency of task performance.

One goal of current research is to identify the areas of the brain responsible for parsing and interpretation. The areas and physiological events necessary for a function are revealed through the effects of lesions that occur either naturally or experimentally, as in transcranial magnetic stimulation: deficits that follow lesions imply that the lesioned aspect of the brain is necessary for the deficient function, while spared functions that follow lesions imply that the lesioned aspect of the brain is unnecessary for the function. The areas and physiological events that are sufficient for a function are revealed through the study of neural responses to linguistic tasks; areas or physiological events affected by psychological operations are a subset of those sufficient for the function that is required for the performance of those psychological operations. The implementation of these research approaches involves many decisions about methods, analyses, and interpretation of results that are subject to discussion.

The most widely accepted model of neural organization for cognitive processing, including syntactic processing, is localization (Friederici 2002; Grodzinsky 2000): the view that particular syntactic computations are processed in circumscribed areas of the perisylvian association cortex, which are 
defined by cytoarchitectonic or, more recently, receptotectonic criteria. These models do not deny macroscopic variation across individuals with respect to the grossly defined areas of the brain that support a language operation, but rather attribute this variability to variability in the mapping of cytoarchitectonic areas onto grossly defined brain areas, such as gyri (Amunts et al. 1999), and to individual differences in connectivity between areas (Anwander et al. 2007). These models postulate localization of syntactic functions in circumscribed brain regions, when brain regions are correctly described at the cytoarchitectonic, receptotonic, or other cellular or subcellular level. Models of this sort carry the implication that the computational capacities of a brain area are determined by its unique informationally relevant features.

The alternative is that computational capacities are determined by the neurological features that are common to a broader range of brain areas within a specified, but more extended brain region. If that is the case, a functional capacity such as language comprehension or, more narrowly, specific syntactic processes, could be distributed over different areas within the perisylvian cortex more or less evenly or unevenly (e.g., Mesulam 1998), localized in a number of areas that have the critical cellular features but which are otherwise unrelated (so-called "degeneracy"), or variably localized in different individuals (Caplan et al. 2007b). Borrowing a term from Lashley (1950), Caplan (1994; Caplan et al. 2007b) used the term "equipotential" to refer to an initial neural state in which many areas, or a large area, are capable of supporting a syntactic operation and the determination of which part of the potentially responsible neural system ultimately supports any given operation is determined by a variety of factors.

These possibilities have implications for mechanisms that underlie the evolution of the neural substrate for language and syntax. Since, as far as is currently known, all genes that are expressed in language-related cortex are expressed in more than one cytoarchitectonically defined area, invariant localization requires a confluence of genetic effects. On the other hand, initial equipotentiality and ultimate variable localization or distribution of a syntactic operation over a wide area of the brain is compatible with multiple patterns of gene expression resulting in cortex that could support a syntactic operation, coupled with a variety of effects of epigenetic factors, the developmental history of an individual, and other influences (including the presence of disease).

Though they cannot both be correct for a single parsing or interpretive operation, the localized and the distributed/variable models each have their supporters. Support for a localized organization of the brain comes from data that report certain syntactic phenomena to be observed consistently in a specific brain region, such as scrambling in BA 44 or movement in BA 45 (Ben-Shachar et al. 2003; Santi and Grodzinsky 2007b; Friederici et al. 2006a). A particular model of localization can be found in Friederici's position paper (this volume) which claims that two pairs of cytoarchitectonically defined areas of cortex, which are functionally and structurally interconnected via different white matter fiber 
tracts, support specific functions in syntactic computations. According to this hypothesis, one network consists of the deep frontal operculum in the medial IFG and the anterior STG and is responsible for processing local phrase structures. The processing of complex sentences with a hierarchical structure is supported by a second network which consists of Broca's area (in particular BA 44) and the posterior STG. In Friederici's view, activation in these brain areas is not specific to language processing; they are also involved in other cognitive processes. The specific function of these areas for language processing emerges from their contributions to a broader network of perisylvian areas and develops during normal ontogenesis. In the case of a major disturbance, such as lesions of these areas early in development, plasticity of the maturating brain allows other parts of the cortex, especially homologous areas of the contralateral hemisphere, to take over these functions .

Evidence for other models comes from the study of aphasia as well as other neuroimaging results. Caplan (this volume) reviews evidence that strokes in either the posterior or anterior parts of the perisylvian cortex are associated with normal performances on tasks that require syntactic comprehension, indicating that different parts of this area are not necessary for syntactic operations in individual cases. He presents data that the severity of deficits in various aspects of syntactic processing varies greatly in patients whose lesions occupy the same proportion of the perisylvian cortex and that the proportion of the perisylvian cortex that is lesioned in patients with similar degrees of impairment of various aspects of syntactic processing also varies greatly. These results lead to the view that there are individual differences in the areas that are necessary for particular syntactic operations. Caplan et al. (2008) argue that BOLD signal correlates of the same or very similar syntactic contrasts often show multiple areas of activation within a single study and considerable variation across studies.

Hagoort (this volume) argues that a particular cognitive function, such as syntax, is most likely subserved by a distributed network of areas, rather than by a local area alone. A one-area-one-function principle is in many cases not an adequate account of how cognitive functions are neuronally instantiated. Connectivity is the major force in shaping the functional contributions of a particular piece of cortex.

These major disagreements result in part from differences in how the research has been conducted (i.e., choice of methods) and in how the results have been interpreted. Many questions arise about experimental design. The activation of brain areas during certain tasks is inferred on the basis of statistical models that usually employ a subtraction between experimental conditions to isolate a specific process under investigation. The resulting activation pattern refers to only those activations that are stronger in one condition than in the other. Using this approach, activation common to both conditions is invisible, which is intended according to the specific process under investigation. However, the choice of contrasts is critical in both aphasiology and functional 
neuroimaging. Baseline sentences need to test all relevant operations that are antecedent to the parsing and interpretive operations under study. For instance, testing for a deficit in the ability to relate a noun phrase to a particular position in a sentence (i.e., to a trace) requires showing that structures with that feature cannot be comprehended normally, that the patient can understand thematic roles in sentences that do not require the operation (e.g., in active sentences), and that a patient can understand sentences that require relating noun phrases to other referentially dependent items (e.g., reflexives).

Another reason for disagreement is that most neuroimaging studies are done on comprehension (parsing), whereas many patient studies have focused on language production (syntactic encoding). The few imaging studies that have been done on syntactic encoding and on comparing encoding and parsing suggest that in comprehension one might be able to bypass syntax, but not so in production. If there have been evolutionary selection pressures and precursors for language, they are very likely related to wiring up the brain for language production and the role of syntax as an intermediary between a nonlinearized conceptual representation and a linear string of speech sounds. To understand the neurobiology of syntax, it might be worthwhile to shift the balance from comprehension to syntactic encoding in language production.

With respect to what has yet to be done, many basic studies are needed to shed light on the neural organization for syntactic processing. On the psychological side, it is becoming apparent that, in both aphasiology and functional neuroimaging, parametric variation of a factor that affects an operation or repetition suppression paradigms are designs that have much promise, but they are only beginning to be used. Qualitative contrasts, which are more commonly used, are hard to interpret. In both aphasiology and functional neuroimaging, the study of the effects of a syntactic contrast in more than one task is critical, but very few studies examine task effects on syntactic determinants of aphasic performance or neural activity in normal subjects.

In addition to these issues, there are several sources of individual variability in functional neuroimaging studies other than distribution, duplication, or individual differences of the areas that support syntactic processing. These sources of differences across studies include variance in the statistical methods used, normalization algorithms, the effects of the tasks used upon syntactic processing, differences in task performance when participants process different sentence types, differential use of ancillary (sometimes strategic) cognitive mechanisms such as subvocal rehearsal in different sentence types, and others. There are differences in parsing and interpretive processes as a function of language, which raises the possibility that different patterns seen in different languages might reflect invariant localization of different syntactic and/or parsing operations. Thus, understanding the reasons for variable results in different studies requires detailed examination of methods, and progress can be expected as this examination reveals limitations of existing methods, leading to better and more detailed experiments. Other analyses of existing data may also be useful, 
such as conjunction analyses of the effects of particular contrasts over tasks, languages and participant groups, meta-analysis of existing studies.

The extent of individual variability in the neural organization for syntactic processing remains an open, basic question. It is worth noting that variability is very common at every level of biology. Phenotypic variation results from genetic, environmental, and developmental variation. Thus, even in genetically identical individuals who grow up in the same environment, some phenotypic variation can be found due to subtle differences in the factors that affect the individuals during development. Thus, the reasons for the various results found in different studies constitute an important area for future study.

\section{Differences between Languages}

Friederici proposed that, in general, the same brain areas may be recruited for different tasks depending on the functional significance of different linguistic markers. One crucial example is thematic role assignment, which seems to be done by the LH, but the cues used for this computation might differ greatly across languages. Investigating different cues across languages, Bornkessel and Schlesewsky (2006) argued that Broca's area supports thematic role assignment independent of the particular cue (word order, case marking, or animacy). They suggest that this region is responsible for mapping the linear sequence of cues regarding the position of a noun on a thematic hierarchy onto the thematic role assigned to each noun.

The idea that areas of the $L H$ become responsible for the efficient use of whatever cue is most reliable or available in a language is supported by developmental studies. Studies by Slobin and Bever (1982), MacWhinney et al. (1985), and Pléh $(1989,1990)$ reveal interesting and intricate differences between languages in the use of different structural cues in agent assignment, such as case marking or word order. Interesting temporal developmental differences follow: In Turkish, due to its clear case marking (the accusative is coded by vowels), children become proficient in agent assignment ("who-did-whatto-whom") by 2 years of age. Hungarian children also use case marking, but it is less transparent in this language; thus, children are tuned to the use of exclusive case marking around 31/2 years of age, whereas English-speaking children stabilize the order-based strategy around the age of 5 . In Serbo-Croatian, where there is an interaction between animacy and case marking, children use a combined word-order and case-marking strategy to assign thematic roles.

In English, Bever (1971) showed that the increase of effective LH dominance in language processes correlates with increasing use of the "first noun is the agent" strategy in sentence interpretation. In Hungarian, Pléh (1982) showed that children of the same age (4-6 years) displayed the opposite tendency; namely that with the increase of LH lateralization, a decrease was observed in the use of order-based strategies. It seems to be that the LH is tuned to the most valid cues of the given language. Therefore, the same brain areas 
recruited to support order-based strategies in English support morphologybased strategies in Hungarian.

Another much studied example is the domain of prosody. Suprasegmental intonational patterns are mainly represented and computed by the RH (Meyer et al. 2002) whereas in tonal languages, where (Gandour et al. 2002; Gandour and Dardarananda 1983) prosodic cues (i.e., tones) have lexical consequences, these are computed and represented by the LH.

Clearly, there is a need to do systematic cross-linguistic comparisons using identical methods, both behaviorally and in regard to neuroscience data gathering, to reveal the exact nature of neural network activation in typologically different languages.

\section{Other Brain Features: Receptor Structure}

Theories of regional specialization raise many issues that must be considered from both a psychological and neural perspective. The most fundamental is: What is the proper decomposition of function and structure? We have briefly discussed what we consider to be important aspects of syntactic representations (for further discussion, see Tallerman et al., this volume). From a neural perspective, we need to go beyond both macroscopic landmarks and cytoarchitectonic classifications, such as that of Brodmann (Figure 14.3), to identify brain areas that could be related to syntax.

Receptor structure is a good candidate for future research. In addition to the most widely distributed major neuroreceptors and neurotransmitters (glutamate, GABA-benzodiazepine, monoamines, acetylcholine, endocannabinoid, opioid), there are several hundred known neuroreceptors as well as neurotransmitter systems in the primate brain. The fine balance between these systems (i.e., the brain's receptor fingerprint) is determined by genetic and epigenetic factors, as well as by ongoing brain activities and the brain's interactions with its social or physical environment. Individual cortical areas have typical receptor structures which are usually characteristic for each cytoarchitectonically defined area (Brodmann area; see Figure 14.4) (Zilles et al. 1991, 2002, 2004; Amunts et al. 1999; Amunts and Zilles 2001; Eickhoff et al. 2007a, b).

The human brain's receptor fingerprint is changing continuously, in response to continuous challenges, including normal or physiological challenges (maturation, aging, habituation, sensory processes, social interactions, diurnal rhythms, etc.), pathological challenges (schizophrenia, depression, epilepsy, etc.), internal (endogenous neurotransmitter release due to, e.g., thinking or meditation) or external pharmacological challenges (medicines, drug abuse). The human brain's receptor fingerprint has enormous individual variations and thus could underlie variability in regional specialization. At the same time, the human brain's receptor fingerprint shows a close correlation with personality types, behavioral traits, and temperament. For instance, the level of dopamine shows a close correlation with extroverted or introverted behavior or 
(a)

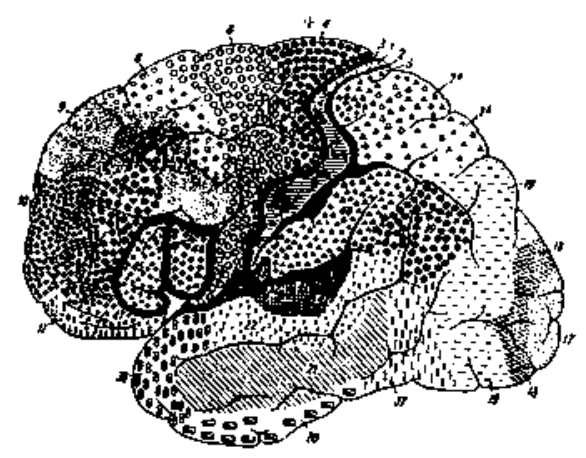

(b)

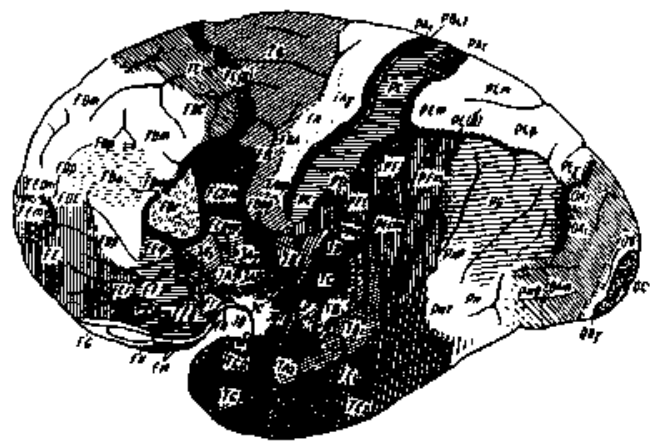

(c)

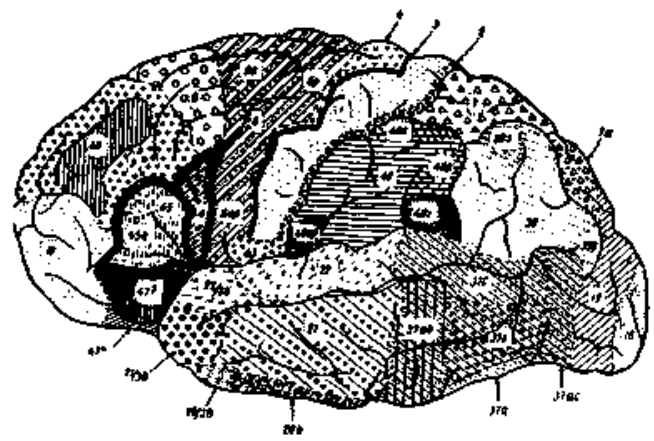

Figure 14.3 Cytoarchitectonic classification of brain areas by Brodmann (reprinted with permission from Amunts et al. 1999).

with novelty seeking, whereas serotonin correlates with harmony or challenge seeking, with proneness to transcendental experience, or depression (Gulyás 2007; Farde et al. 1997; Zald et al. 2008).

Thus receptor profiles could contribute to stable neural systems that underlie syntactic processing. An open issue is whether the involvement of the perisylvian cortex in syntactic processing might be based on certain properties in its receptor architecture. To date, it is unclear whether receptor 


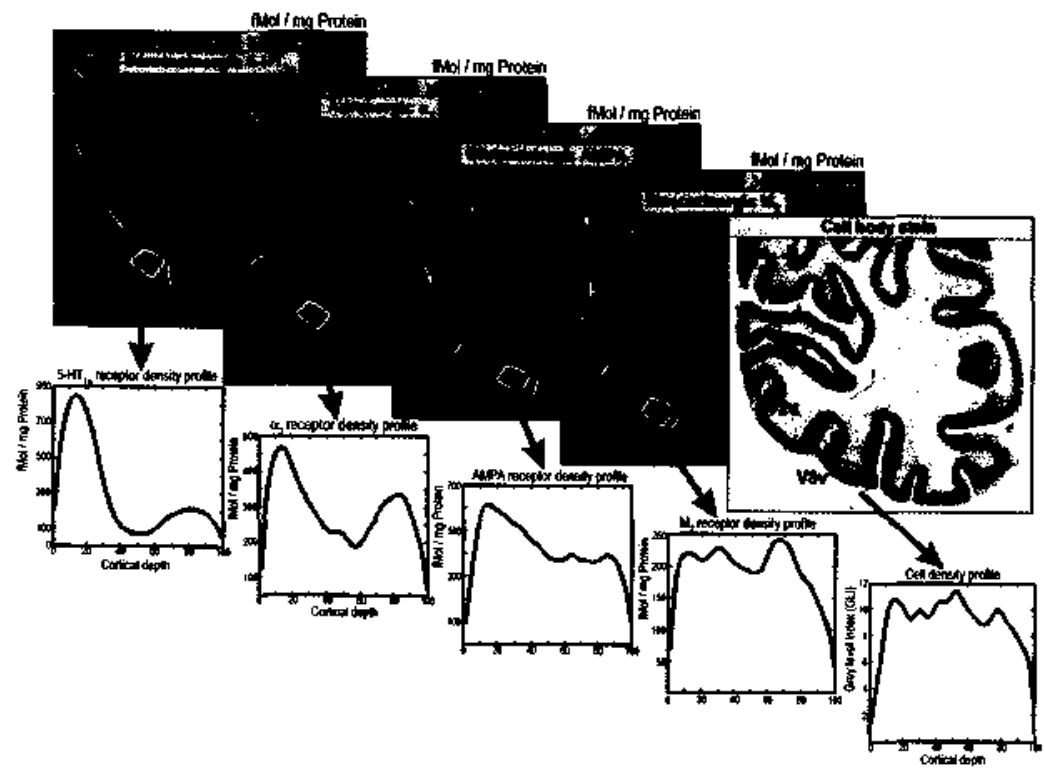

Figure 14.4 Receptor structure of brain areas (after Eickhoff et al. 2007b).

architecture is a precondition or a consequence of shared functionality between two brain areas.

At the same time, many crucial aspects of morphology, laminar distribution, and synaptic targets are very well conserved between areas and between species (Douglas and Martin 2004). Functional differences between brain areas might, therefore, be mainly due to variability of the input signals in forming functional specializations. Domain specificity of a particular piece of cortex might thus not so much be determined by heterogeneity of brain tissue, but rather by the way in which its functional characteristics are shaped by the input. Moreover, findings of neuronal plasticity (e.g., the involvement of visual cortex in verbal memory in the congenitally blind; Amedi et al. 2003), suggest substantial plasticity in structure-to-function relations (see Hagoort, this volume).

\section{The Temporal Dimension of Syntactic Processing}

Electrophysiological measures provide data of the temporal aspects of brain mechanisms underlying syntactic processing. The most intensely studied ERP components, often studied using violation paradigms and by presenting sentences with increased processing difficulty, are briefly described here.

Based on the time course of language-related ERP effects, one can say that the major syntactic and semantic processing events happen between 150 and 800 milliseconds. In connection with syntactic processes, two classes of 
syntax-related ERP effects have been consistently reported over a period of more than ten years. Examples of sentence material that would elicit the four ERP components are:

$\begin{array}{ll}\text { (4) (a) *The boy of eats ice cream } & \text { ELAN, P600 } \\ \text { (b) *The boys eats ice cream } & \text { LAN, P600 } \\ \text { (c) The boy eats socks } & \text { N400 }\end{array}$

One type of ERP effect related to syntactic processing is the P600 (Hagoort et al. 1993; Osterhout and Holcomb 1992). P600 is reported in relation to syntactic violations, syntactic ambiguities, and syntactic complexity. This effect occurs in a latency range between roughly $500-800 \mathrm{~ms}$ following a lexical item that embodies a violation or a difference in complexity. However, the latency can vary, and earlier P600 effects have also been observed (Hagoort 2003; Mecklinger et al. 1995). Another syntax-related ERP is a left anterior negativity, referred to as LAN or, if earlier in latency than $300-500 \mathrm{~ms}$, as ELAN (Friederici et al. 1993). In contrast to the P600, (E)LAN has thus far been (almost) exclusively observed in syntactic violations. LAN is usually observed within a latency range of $300-500 \mathrm{~ms}$; ELAN is earlier, with an onset between 100-150 ms. The topographic distribution of ELAN and LAN is very similar. The most parsimonious explanation is, therefore, that the same neuronal generators are responsible for LAN and ELAN, but the temporal profile of their activation varies (for an alternative view, see Friederici, this volume). A negativity around $400 \mathrm{~ms}$ (N400) with a central topography is related to semantic processes, for example, at semantic violations and corresponding lexical search processes.

ERP data provide an example of the feedback from neurological studies to models of language processing. One of the most remarkable characteristics of speaking and listening is the speed at which it occurs. Speakers easily produce 3-4 words per second; information that has to be decoded by the listener within roughly the same time frame. Considering that the acoustic duration of most words is in the order of a few hundred milliseconds, the immediacy of the ERP effects is a highly salient feature. The ELAN has an onset of 100-150 $\mathrm{ms}$, the onset of the N400 and the LAN is approximately at $250 \mathrm{~ms}$, and the P600 usually starts at about $500 \mathrm{~ms}$. The majority of these effects happen well before the end of a spoken word. Classifying visual input (e.g., a picture) as coming from an animate or inanimate entity takes the brain approximately 150 $\mathrm{ms}$. If we use this as our reference time, the early brain response reflected in the ELAN to a spoken word is remarkable, to say the least. In physiological terms, it might be just too fast for feedback to have an effect on parts of primary and secondary auditory cortex involved in first-pass acoustic and phonological analysis. This suggests that what happens in online language comprehension is substantially based on predictive processing. Under most circumstances, there is just not enough time for top-down feedback to exert control over a preceding bottom-up analysis. Very likely, lexical, semantic, and syntactic cues are used 
to predict characteristics of the next upcoming word, including its syntactic and semantic makeup. A mismatch between contextual prediction and the output of bottom-up analysis results in an immediate brain response activating additional processing resources used in revising the products of the initial online interpretation process (see Hagoort, this volume).

\section{Neural Organization for Processing Syntax in Children and Individuals with Impaired Development}

Apart from the individual variability in performance and, possibly, the neural substrate for syntactic processing that can be found in healthy, monolingual adults with a normal ontogenetic background, it is important to investigate how great the divergence can be in individuals whose language systems differ from that of adult monolinguals, such as children, bilinguals, and individuals who have experienced brain damage early in their development. Studies of these groups suggest that different brain areas can be recruited during ontogeny to support language, with similar efficiency to that seen in adult monolinguals.

\section{Children}

Early investigations of language acquisition in children by functional brain imaging focused mainly on language lateralization during rather broad task requirements, such as word generation or text comprehension (Gaillard et al. 2001; Lee et al. 1999). Results of these early experiments showed that children make use of the same network of perisylvian cortical areas in the inferior frontal and superior temporal cortices during language processing as adults. However, in some studies, the organization of language in the developing brain seemed to be less left-lateralized than in adults (Brauer and Friederici 2007, Gaillard et al. 2000; Holland et al. 2001; Ulualp et al. 1998); other studies did not find any differences in language laterality between adults and children (Balsamo et al. 2006; Lohmann et al. 2005). The degree of lateralization appears to depend on age, task, and cortical area (Holland et al. 2007). Nevertheless, the developing language comprehension system shows some hemispheric specialization for certain aspects of language: e.g., the specialization of the $\mathrm{RH}$ for prosodic information (Meyer et al. 2003) was observed in young children (Wartenburger et al. 2007) and infants (Homae et al. 2006).

With respect to the intra-hemispheric organization for language, children show less neural specification than adults (Brauer and Friederici 2007). Using a violation detection task with its attendant limitations, Brauer and Friederici (2007) found that children used the entire perisylvian network during sentence comprehension, as opposed to the regional specialization for syntactic and semantic processes seen in adults. A specification for syntactic processes was observed only in Broca's area in the IFG, where there was more activity 
associated with syntactic processing than is seen in adults. Interestingly, this is the same area that is also more strongly engaged in adult second language learners and in adult native speakers when processing more complex sentence structures. The inference is that this pronounced involvement of the IFG most probably reflects higher processing demands (Bomkessel et al. 2005; Rüschemeyer et al. 2005).

With respect to the timing of syntactic and semantic processes during sentence comprehension, electroencephalic (EEG) investigations in very young children have shown ERPs that resemble those seen in adults during syntactic processing (ELAN, P600) and during semantic processing (N400). These ERPs components are, however, slightly later and more sustained (Friedrich and Friederici 2004, 2005; Oberecker and Friederici 2006; Oberecker et al. 2005).

The anatomical bases for differences in intra-hemispheric organization for syntactic processing in children and adults have begun to be explored. Connectivity through the subcortical white matter connections revealed by diffusion tensor imaging shows differences between adults and children in fiber tract integrity in exactly those perisylvian regions where the functional differences between them are observed (i.e., IFG and STG) (Brauer et al. 2008). Presumably, this is based on lower myelination of these fiber pathways in the immature brain (Paus et al. 1999). The main fiber tract connecting IFG and STG shows continuous maturation during development until adolescence (Giorgio et al. 2008). Other white matter connections, such as those underlying the sensory and motor cortices, mature much earlier than those in temporal language areas, arguing for a particularly slow maturation of language-related pathways (Pujol et al. 2006). During maturation, increasing integrity and myelination of white matter fiber tracts permits faster and more accurate information transmission between the cortical structures involved in the network of language comprehension. Simultaneously with changes in white matter, gray matter maturation progresses during childhood and adolescence.

Thus, the development of the human brain is accompanied by a general pattern of progressive and regressive adjustments, including cortical and subcortical brain structures (Toga et al. 2006). Brain maturation is characterized by changes in gray matter with a reduction of cortical thickness and density (Giedd et al. 1999; Sowell et al. 2003). Pruning out of synapses and reduction of neuropil might be responsible for this (Staudt et al. 2000). Simultanuously, white matter gain occurs due to ongoing myelination of the fiber pathways, a process which lasts until young adulthood (Barnea-Goraly et al. 2005; Lebel et al. 2008). These maturational processes are likely to be related to, if not underlie, functional development which, in turn, might affect structural manuration.

The study of the neural basis for syntactic processing in children is linked to the question of the neural basis of the capacity to leam a syntactic system that has features found in human languages. Clearly, only learning systems with particular properties, likely coupled to cognitive systems with particular properties, are able to acquire natural language syntax, and these learning and 
related cognitive systems must have evolved in humans. Behavioral studies have made claims about these learning and cognitive systems on the basis of the study of syntax acquisition in children. Findings such as those presented above are relevant to the ontogenetic neural changes that lead to changes in the nature and efficiency of syntactic processing, but only provide very general information about the features of neural systems that have evolved in such a way so as to allow syntax to be acquired. A number of studies of adults leaming syntactic systems have, however, been interpreted as providing more information on this topic. Opitz and Friederici (2004), for example, demonstrated that learning a grammar is initially supported mainly by the hippocampus and that, as learning proceeds, the activation in the hippocampus decreases while activation in Broca's area increases. Studies by Tettamanti et al. (2002) have been interpreted as showing that Broca's area is responsible for the ability to apply principles of Universal Grammar to leaming artificial languages, but this interpretation has been questioned (Caplan 2007b).

We do not know the minimum amount of input that is required for a child to be able to leam a language without being negatively affected. However, it seems that it is much less than is usually expected. Surprising examples include hearing children with deaf parents for whom acoustic input is confined to the media and occasional encounters with relatives and yet still develop good language skills. Conversely, deaf children who communicate with their parents using a home-style signing language can readily learn real, grammatical sign language when going to school; even without proficient teachers, they develop grammatical signing themselves. Similarly, in groups of children who hear only pidgin at home, Creole languages emerge.

A well-established aspect of language development is that it is usually less efficient after a certain age-the "critical period." The critical period for language acquisition seems to differ for different components of language (Hakuta et al. 2003). The reasons why language cannot be perfectly acquired after this critical period are not well understood and may be due to several mechanisms. It has been shown that long-distance growth of axons is not possible after a certain age. Most pruning also takes place at an early age; thereafter plasticity may even decrease (Huttenlocher 2002). Myelination may also play a role, as discussed earlier.

\section{Individuals with Impaired Development}

The effects of lesions on the areas that support syntactic processing reveal additional aspects of neural organization for these processes. Before a certain age, focal damage to LH language regions does not necessarily lead to impaired linguistic capacities in the adult state (Bishop 1988; Bates 1999). In epileptic children, where Broca's area has been damaged, the center of language production "moves" to the RH homologue of that area, and these children exhibit good linguistic abilities, although language development is 
slow (Vargha-Khadem et al. 1997). These new areas in the RH are also often activated in unaffected individuals during language tasks, although their particular functions are not yet known. The importance of RH has also been emphasized by Bates et al. (1997), who examined children that suffered brain injury to either the $\mathrm{LH}$ or RH before 6 months of age. Results from 10-17 months suggest that children with RH injuries are at greater risk for delays in word comprehension and in the gestures that normally precede and accompany language onset. The relevance of the RH during early childhood might reflect the fact that early language development is based to a large extent on prosodic processes represented in the $\mathrm{RH}$. Likewise, following early surgical interventions in the LH, RH activity can be observed in linguistic tasks but not entirely over homologue areas. Using ARI, Liegeois et al. (2004) showed that the age of lesion effects the neural basis for language: following early LH damage, the RH takes over linguistic functions, but if the damage occurred after the age of 5 , language remains based in the LH.

\section{Potential Relations between Nonlinguistic Functions and Syntax}

One hypothesis of how syntactic capacities evolved is that they are due to exaptations: brain areas that evolved under selection pressures for other functions became capable of supporting syntactic processing. Several hypotheses of this sort have been suggested.

By modernizing the classical gestural theory of the origin of language, Corballis emphasizes that modern spoken language was modeled on simple elementary gesturing (Corballis 2002). He proposes that autonomous speech may have arisen only as recently as 50,000 years ago, shortly before the cultural explosion. Spoken language allowed humans to use their hands for technology, which led to the development of more sophisticated cultural products.

The discovery of mirror neurons (neurons that fire when someone performs a specific action as well as when they observe someone else performing the same action) in the ventral premotor cortex has led to theories about potential links between motor gestures and language. Mirror neurons provide a direct link between sender and receiver by which parity (what counts for the sender of a message also counts for the receiver) and direct comprehension becomes possible (Rizzolatti and Arbib 1998). Parity is seen as a key to the initiation of language evolution.

Other capacities have also been suggested as evolutionary precursors of syntax. One is a nonlinguistic basis for representing events. When speakers of separate languages that differ in their predominant word order are asked to describe an event by using gestures without speech, they uniformly use a fixed actor-patient-act order (e.g., girl-box-cover; Goldin-Meadow et al. 2008). This order, which is analogous to the subject-verb-object pattern found in many languages of the world, is also the order found in the earliest stages of 
newly evolving (gestural) languages. This observation suggests that when humans initially created language, they may have exploited a natural disposition for presenting events nonverbally.

Social and internal cognitive capacities may also be a precursor of syntactic abilities. They are believed to have provided adaptive pressures for further evolution of capacities such as anticipation and working memory because language enriches the ability to plan for the future (Arbib 2008). The ability to "plan ahead" is seen in many nonlinguistic functions, such as motor planning (e.g., as in animal foraging). An action like pushing a door, for example, is a function of situations where the door is closed (or open) to situations where it is open (or closed). A plan to push a (closed) door, and then go through it, is the composition of two such functions:

"Push, then go through."

Such a plan is already (weakly) hierarchical and the definition of planning itself is recursive, thus a plan is an elementary action, or a plan is the composition of two plans. "Plans" of nested relations might therefore have preceded and triggered the evolution of language.

Hierarchical computations involved in motor planning and language do not have to be supported by the same brain regions because the aptitude for such computations may not be restricted to one specific region in the brain. Hence, while Broca's area (BA 44/45) is involved in hierarchically organized sentence processing or in the processing of artificial grammar (Friederici et al. $2006 \mathrm{~b}$; Bahlmann et al. 2008), processing hierarchical structures of nonlinguistic nonsense shapes, seemed to involve the pre-supplementary motor area in addition to Broca (Bahlmann et al., submitted). It is worth noting that the majority of studies that investigated manipulation of sequential information documented the involvement of Broca's area independently of the nature of information that was manipulated. Gelfand and Bookheimer (2003) showed that the posterior portion of Broca's area responded to sequence manipulation tasks, independent of whether the stimuli were composed of phonemes or hummed notes. Similarly, the invention of novel motor sequences in musical improvisation recruits a network of brain regions that includes Broca's area (Berkowitz and Ansari 2008). Ullman (2004), therefore, suggested that the rule-governed combination of lexical items into complex representations evolved from a network of frontal, basal ganglia, parietal, and cerebellar structures, which supports the learning and execution of any skills that involve sequences.

Though planning abilities may be one evolutionary precursor for language, some aspects of language, including features of syntax, appear to have a more complex structure than planning in animals. An example is long-distance dependency of the kind exhibited by the relative clause construction. A person who has the goal state of having a banana, and constructs a plan: 
(6) GRASP(GO_THROUGH(PUSH))

"Push, then go through, then grasp."

which is applied to a banana on the other side of a door, has in some sense established a long-distance (three-step) dependency between the present state and the banana.There is some evidence, however, that animal planning does not have this character. Animal planning is more like reactive search through reachable situations (Koehler 1925), and in that sense is probably expressible by finite-state machines. Most likely, the intrinsically recursive character of language has other origins. One that has been suggested is the additional distinctively human involvement of propositional attitude verbs, and the nature of the associated specifically recursive concepts of other minds (Tomasello 1999).

\section{Phylogenetic Development of the Human Brain and the Evolutionary Neural Basis for Syntax}

Language is a recent achievement, but not as recent as other cognitive capacities of the brain, such as arithmetic, reading and writing, for which there has been no time for selection to drive genetic evolution. In contrast to these late cultural innovations, language has been around much longer, making it likely that some genetic evolution has accompanied language evolution. If so, it is legitimate to ask how genes can influence our language faculty by affecting the brain. What is the novelty in the brain compared to that of apes that allows us to handle language?

Increased brain size and specialized brain areas are two commonly cited features of the brain that may be the basis for this capacity. However, there are problems with both explanations. Microcephalics suffer from several cognitive deficits, but they are usually still much better at language than nonhuman primates (Woods 2005), arguing that increased brain size cannot be the (only) evolutionary change in the brain that led to language. We should also bear in mind that there is great variation in brain size within the general population. By the same token, early lesions of the LH can be compatible with the development of good language, suggesting that the emergence of specific neural areas is also not the sole basis for language evolution. Taken together, these facts suggest that the human brain is able to represent and process language, albeit with less efficiency, even when it falls outside of the normal range of brain size and has encountered major changes in the areas normally involved in language functioning. We do not know what the computational correlates of these differences are, but we can pose the following inferences: Synaptic dynamics, functional connectivity between cells and between cortical regions based on fiber tract connections, propagation of spike packets can, in principle, all be crucially different. In particular, the immature human brain could have a widespread latent capacity to handle operations on hierarchical 
structures, which is an empirically testable question. This is compatible with the statement that in normal brains, Broca's area is in fact specialized for some syntactical operations.

One possibility is that the emergence of language and syntax depended upon the evolution of a greater capacity for neural specialization during ontogeny than was found in nonlinguistic ancestors (see Szamado et al., this volume). Accordingly, an "equipotential" initial state (in the sense of the word used above) would be transformed into one of a number of adult specializations, such as a specialization of Broca's area for some syntactical operations. In the young human brain, this specialization could be more quantitative than qualitative. In contrast, in the mature brain, the difference becomes qualitative due to ontogenetic progressive modularization. Changes in the extent to which this ontologenetic specialization can occur may be one step in the evolution of a brain that supports language and syntax. We note that this suggestion places less importance on phylogenetic analogies to language development during ontogeny than the view that the evolution of language, or syntax, requires highly specific, human cortical microcircuitry. The idea of ontogenetic maturation repeating phylogeny is hard to evaluate, since many changes in brains across species (e.g., brain size, the relative amount of white matter, functional and anatomical connectivity) are correlated. Qualitative differences could be an emergent property of quantitative changes (as in phase-transitions in physics).

\section{Acknowledgments}

Our discussion benefitted from the participation of many others at this Forum. The contributions of Derek Bickerton, Dorothy Bishop, Terrence Deacon, Tom Givón, James Hurford, Frederick Newmeyer, Luigi Rizzi, Mark Steedman, Luc Steels, Eörs Szathmáry and Stephanie White are specifically acknowledged here. 